Pacific Journal of Mathematics

COMPUTATIONS OF THE MULTIPLICITY FUNCTION 


\title{
COMPUTATIONS OF THE MULTIPLICITY FUNCTION
}

\author{
S. R. FOGUEL
}

1. Introduction. Let $H$ be a separable Hilbert space. The following two problems will be studied:

1. Given a bounded normal operator $A$, of multiplicity $m$, what are the conditions, on the bounded measurable function $f$, so that the multiplicity of $S=f(A)$ is $n, n<\infty$ ?

2. How to compute the multiplicity of a normal operator that commutes with a given normal operator, of finite multiplicity?

Notation. Let $S$ be a normal operator of multiplicity $n, n<\infty$. There exist a Borel measure $\mu$ and $n$ Borel sets in the complex plane $e_{1} \supset e_{2} \supset \cdots \supset e_{n}$, such that, up to unitary equivalence,

$$
\begin{gathered}
H=\sum_{i=1}^{n} L_{2}\left(\mu, e_{i}\right) \\
S\left(\begin{array}{c}
f_{1}(\lambda) \\
\vdots \\
f_{n}(\lambda)
\end{array}\right)=\left(\begin{array}{c}
\lambda f_{1}(\lambda) \\
\vdots \\
\lambda f_{n}(\lambda)
\end{array}\right)
\end{gathered}
$$

This is the Multiplicity Theorem. (See Theorem X. 5.10) of [1]. The operator $S$ has uniform multiplicity if $e_{1}=e_{2}=\cdots=e_{n}$.

The resolution of the identity, of a normal operator $A$, will be denoted by $E(A ; \alpha)$. The Boolean algebra of projections, generated by

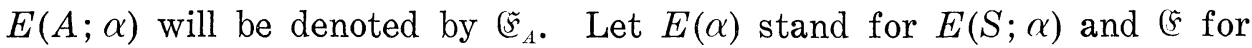
$\mathfrak{F}_{s}$. Throughout this note all operators are assumed to be bounded.

We shall use the following results from [2]:

Let $S$ be a normal operator of multiplicity $n$, and $B$ a normal operator that commutes with $S$. Let $H$ and $S$ be represented by 1.1 .

THEOREM A. There exist $k$ Borel measurable bounded complex functions $y_{1}(\lambda), \cdots, y_{k}(\lambda)$ and $k$ matrices of Borel measurable bounded complex functions $\varepsilon_{1}(\lambda), \cdots, \varepsilon_{k}(\lambda)$ such that:

For a fixed $\lambda$ the matrices $\varepsilon_{i}(\lambda)$ are disjoint self adjoint projections whose sum is the identity and

$$
B\left(\begin{array}{c}
f_{1}(\lambda) \\
\vdots \\
f_{n}(\lambda)
\end{array}\right)=\left(\sum_{i=1}^{k} y_{i} \varepsilon^{i}(\lambda)\right)\left(\begin{array}{c}
f_{1}(\lambda) \\
\vdots \\
f_{n}(\lambda)
\end{array}\right) .
$$

Received October 21, 1958, and in revised form April 24, 1959. This work has been partially supported by the National Science Foundation. 
Equivalently, if the self adjoint projections $E_{i}$, are defined by

$$
E_{i}\left(\begin{array}{c}
f_{1}(\lambda) \\
\vdots \\
f_{n}(\lambda)
\end{array}\right)=\varepsilon_{i}(\lambda)\left(\begin{array}{c}
f_{1}(\lambda) \\
\vdots \\
f_{n}(\lambda)
\end{array}\right)
$$

then

$$
\left\{\begin{array}{l}
B=\sum_{i=1}^{k} y_{i}(S) E_{i} \\
E(B ; \alpha)=\sum_{i=1}^{k} E\left(y_{i}^{-1}(\alpha)\right) E_{i} .
\end{array}\right.
$$

REMARK. In the above decomposition the numbers $y_{i}(\lambda)$ for a fixed $\lambda$ are different eigenvalues of a certain matrix. Thus for each $\lambda$ there is an integer $k^{\prime} \leq k$ such that

$$
y_{i}(\lambda) \neq y_{j}(\lambda) \quad i \neq j \quad i, j \leq k^{\prime}, \quad \varepsilon_{i}(\lambda) \neq 0 \quad i \leq k^{\prime},
$$

and

$$
\begin{aligned}
y_{k^{\prime}+1}(\lambda) & =\cdots=y_{k}(\lambda)=0, \\
\varepsilon_{k+1}(\lambda) & =\cdots=\varepsilon_{k+1}(\lambda)=0 .
\end{aligned}
$$

This is essential for the proof of Lemma 2.1. Also the matrices $\varepsilon_{i}(\lambda)$ are $n \times n$ matrices.

THEOREM B. The number $n$ is the largest integer such that there exists a nilpotent operator, commuting with S, of order n. See [2] Theorem 3.1 and its corollary.

2. The multiplicity of a function of an operator. The main result in this section is:

THEOREM 2.1. Let $A$ be a normal operator of multiplicity $m$, $m<\infty$, and $f$ a bounded measurable function. The operator $S=f(A)$ has finite multiplicity, if and only if, there exist $k$ disjoint Borel sets $\beta_{1}, \cdots, \beta_{k}$ and $k$ bounded measurable functions $z_{1}(\lambda), \cdots, z_{k}(\lambda)$ such that:

$$
\begin{aligned}
& \text { a. } \sigma(A)=\bigcup_{i=1}^{k} \beta_{i} . \\
& \text { b. if } \lambda \in \beta_{i} \text { then } z_{i}(f(\lambda))=\lambda \text { almost }
\end{aligned}
$$

everywhere, with respect to $E(A ; \alpha)$.

Proof of sufficiency of conditions $a$ and $b$. Let $S_{i}$ and $A_{i}$ be the restrictions of $S$ and $A$ to $E\left(A ; \beta_{i}\right) H$. Then 


$$
S_{i}=\int_{\beta i} f(\lambda) E(A ; d \lambda)
$$

hence

$$
z_{i}\left(S_{i}\right)=A_{i}
$$

Now, it follows from Theorem B that

$$
m u A_{i} \geq m u S_{i} \quad(m u T=\text { multiplicity of } T)
$$

But the multiplicity function is subadditive:

$$
m u S \leq \sum_{i=1}^{k} m u S_{i} .
$$

To see this we have to observe that $m u S$ is the smallest number $n$ such that there exists a set of $n$ elements, $\left\{x_{1}, \cdots x_{n}\right\}, x_{i} \in H$ and span $\left\{E(\alpha) x_{i}, \alpha\right.$ a Borel set $\}=H . \quad(n$ generating elements. $)$

Thus

$$
m u A \leq \sum_{i=1}^{k} m u S_{i} \leq \sum_{i=1}^{k} m u A_{i} \leq m k<\infty
$$

In order to prove necessity we need the following:

LEMMA 2.1. Let $S=f(A)$ have finite multiplicity $n$ and let

$$
A=\sum_{i=1}^{k} z_{i}(S) E_{i}
$$

be the representation 1.3 then $E_{i} \in \mathfrak{F}_{A}$.

Proof. For every Borel set $\alpha E(\alpha) \in \xi_{A}$ because $S=f(A)$. Let $E(\alpha)$ be maximal with respect to the property that $E(\alpha) E_{1} \in \mathfrak{F}_{A}$. Such a maximal projection exists by Zorn's Lemma. Now if $E(\sigma(S)-\alpha) \neq 0$ there exists, by the proof of 3.2 in [2] a set $\beta$ such that:

$$
\beta \subseteq \sigma(S)-\alpha \quad E(\beta) \neq 0
$$

and for some Borel set $\gamma$

$$
E(\beta) E_{1}=E(\beta) E(A ; \gamma) \in \mathfrak{F}_{A} .
$$

This contradicts the maximality of $\alpha$, hence $E(\alpha)=I$.

Proof of necessity of conditions $a$ and $b$. Let $S$ hsve finite multiplicity $n$. By Lemma 2.1 there exist $n$ sets $\beta_{i}$ such that $E\left(A ; \beta_{i}\right)=E_{i}$. Thus 


$$
E\left(A ; \beta_{i}\right) E\left(A ; \beta_{j}\right)=0 \text { if } i \neq j
$$

and

$$
\sum_{i=1}^{k} E\left(A ; \beta_{i}\right)=I \text {. }
$$

Therefore the sets $\beta_{i}$ can be chosen to be disjoint and satisfy condition a. Also

$$
A=\sum_{i=1}^{k} z_{i}(S) E_{i}=\sum_{i=1}^{k} z_{i}(f(A)) E\left(A ; \beta_{i}\right)=\sum_{i=1}^{k} \int_{\beta} z_{i}(f(\lambda) E(A ; d \lambda) .
$$

Hence, if $\beta \subset \beta_{i}$ then

$$
E(A ; \beta) A=\int_{\beta} \lambda E(A ; d \lambda)=\int_{\beta} z_{i}(f(\lambda)) E(A ; d \lambda)
$$

or: on the set $\beta_{i} \lambda=z_{i}(f(\lambda))$ almost everywhere with respect to the measure $E(A ; \alpha)$.

Definition. The function $f$ will be said to have $k$ repetitions, with respect to the measure $E(A ; \alpha)$, if conditions a and $b$ of Theorem 2.1 are satisfied.

In the rest of this section we compute $m u S$. It is enough to consider the case where the operator $A$ has uniform multiplicity $m$ : otherwise $A$ can be written as direct sum of operators of uniform multiplicity and one has to study each component of $A$ separately.

The following Theorem is needed:

THeOREM 2.2 Let $H$ be the direct sum of the orthogonal subspaces $H_{1}, \cdots, H_{k}$. Let $S_{i}$ be a normal operator, on $H_{i}$, of uniform multiplicity $m_{i}$ and $S$ be the direct sum of $S_{i}$.

If

$$
E(S ; \alpha)=0 \text { whenever } E\left(S_{i} ; \alpha\right)=0 \text { for some } i
$$

then

$$
m u S=\sum_{i=1}^{k} m_{i}
$$

Proof. It is enough to prove that $m u S \geq \sum_{i=1}^{k} m_{i}$. Let $\sigma=\sigma\left(S_{1}\right)=$ $\cdots=\sigma\left(S_{k}\right)=\sigma(S)$. By the Spectral Multiplicity Theorem each operator $S_{i}$ can be described as follows: There exists a measure $\mu_{i}$ on $\sigma$ and $H_{i}$ is the direct sum of $m_{i}$ spaces $L_{2}\left(\mu_{i}\right)$. The operator $S_{i}$ is given by

$$
S_{i}\left(\begin{array}{c}
f_{1}(\lambda) \\
\vdots \\
f_{m_{i}}(\lambda)
\end{array}\right)=\left(\begin{array}{c}
\lambda f_{1}(\lambda) \\
\vdots \\
\lambda f_{m_{i}}(\lambda)
\end{array}\right) .
$$


Now, the measures $\mu_{i}$ are equivalent, by the condition of the Theorem. Thus there exist functions $\varphi_{i}, \varphi_{i} \in L\left(\mu_{i+1}\right) 1 \leq i \leq k-1$ such that

$$
\mu_{i}(e)=\int_{e} \phi_{i}(\lambda) d \mu_{i+1}
$$

for every Borel set e. (Radon Nikodym Theorem, see [3], p. 128). Let us define an operator on $H$ :

If $x \in H_{i}$,

$$
x=\left(\begin{array}{c}
f_{1}(\lambda) \\
\vdots \\
f_{m_{i}-1}(\lambda) \\
0
\end{array}\right)
$$

then

$$
M x \in H_{i}, \quad M x=\left(\begin{array}{c}
0 \\
f_{1}(\lambda) \\
\vdots \\
f_{m_{i}-1}(\lambda)
\end{array}\right)
$$

If

$$
x \in H_{i}, \quad x=\left(\begin{array}{c}
0 \\
\vdots \\
0 \\
f_{m_{i}}(\lambda)
\end{array}\right)
$$

then

$$
M x \in H_{i+1}, \quad M x=\left(\begin{array}{c}
\sqrt{\overline{\varphi_{i}(\lambda)} f_{m_{i}}(\lambda)} \\
0 \\
\vdots \\
0
\end{array}\right) .
$$

Where $H_{k+1}$ is the zero space.

It is easy to see that $M$ is a bounded operator and

but

$$
M^{\sum_{i=1}^{k} m_{i}}=0
$$

$$
M^{\sum_{i=1}^{k} m_{i}-1} \neq 0 .
$$


Also $M S=S M$, hence $m u S \geq \sum_{i=1}^{k} m_{i}$.

REMARK. It was proved in Theorem 2.1 that if a function $f$ has $k$ repetitions then

$$
m u f(A) \leq k m u A \text {. }
$$

However the number of repetitions of a function is not uniquely defined. In order to compute $m u f(A)$ we have to find the minimal number of repetitions. This is what the next Theorem does.

THEOREM 2.3. Let $A$ be a normal operator of uniform multiplicity $m$. Let $f$ be a bounded measurable function which has $k$ repetitions with respect to the measure $E(A ; \alpha)$. A necessary and sufficient condition that $m u S=m k$, where $S=f(A)$, is:

There exists a Borel set $\alpha_{0}$

$$
E\left(A ; f^{-1}\left(\alpha_{0}\right)\right) \neq 0
$$

and

$E\left(A ; f^{-1}(\alpha)\right)=0$ whenever $E\left(A ; f^{-1}(\alpha) \cap \beta_{i}\right)=0$ for some $i$ and $\alpha \subset \alpha_{0}$.

Proof. Assume condition 2.1. We may restrict $A$ and $S$ to $E\left(A ; f^{-1}\left(\alpha_{0}\right)\right) H$. Let

$$
H_{i}=E\left(A ; f^{-1}\left(\alpha_{0}\right) \cap \beta_{i}\right) H,
$$

and $A_{i}, S_{i}$ the restriction of $A, S$ to $H_{i}$. Now

$$
f\left(A_{i}\right)=S_{i} \quad z_{i}\left(S_{i}\right)=A_{i}
$$

(See Theorem 2.1.). Thus the operators $S_{i}$ have uniform multiplicity $m$ because the operators $A_{i}$ do. It follows from Theorem 2.2 that the multiplicity of $S$ restricted to $E\left(A ; f^{-1}\left(\alpha_{0}\right)\right) H$ is $m k$. But $m u S \leq m k$, hence $m u S=m k$.

(Note that on $\alpha_{0}$ the operator $S$ has uniform multiplicity $m k$ ). Conversely, let us assume that for each Borel set $\alpha_{0}$ with $E\left(A ; f^{-1}\left(\alpha_{0}\right)\right) \neq 0$, there exists a subset $\alpha$ such that $E\left(A ; f^{-1}(\alpha)\right) \neq 0$ but $E\left(A ; f^{-1}(\alpha) \cap \beta_{i}\right)=0$ for some $i$. Let $E\left(A ; f^{-1}\left(\alpha_{1}\right)\right)$ be maximal with respect to the property

$$
E\left(A ; f^{-1}\left(\alpha_{1}\right)\right) E\left(A ; \beta_{1}\right)=0
$$

Let $E\left(A ; f^{-1}\left(\alpha_{2}\right)\right)$ be maximal, with respect to the property

$$
\alpha_{2} \cap \alpha_{1}=\varphi \text { and } E\left(A ; f^{-1}\left(\alpha_{2}\right)\right) E\left(A ; \beta_{2}\right)=0
$$

and choose inductively $\alpha_{3} \cdots \alpha_{n}, \alpha_{i} \cap \alpha_{j}=\varphi$ 


$$
E\left(A ; f^{-1}\left(\alpha_{j}\right)\right) E\left(A: \beta_{j}\right)=0
$$

There exist such maximal projections by Zorn's Lemma. Now if $E\left(A ; \bigcup_{i=1}^{k} f^{-1}\left(\alpha_{i}\right)\right) \neq I$ there will be a set $\alpha$ and an integer $j$ such that

$$
\alpha \cap\left(\bigcup_{i=1}^{k} \alpha_{i}\right)=0 ; \quad E\left(A ; f^{-1}(\alpha) \cap \beta_{j}\right)=0
$$

Thus $\alpha$, will not be maximal. Let

$$
\bar{\beta}_{j}=\beta_{j} \cup\left(f^{-1}\left(\alpha_{j}\right) \cap \beta_{1}\right), \quad j \geq 2 .
$$

Then $\bigcup_{j=2}^{u} \bar{\beta}_{j}=\sigma(A)$ and on $\bar{\beta}_{j}$ the function $f$ possesses a bounded measurable inverse. Thus $f$ has $k-1$ repetitions and $m u S \leq m(k-1)$.

3. The multiplicity of a matrix of functions. Let $S$ be a normal operator of uniform multiplicity $n$. Let $B$ be a normal operator and $B S=S B$. The operator $B$ is represented as the matrix of functions $\sum_{i=1}^{k} y_{i}(\lambda) \varepsilon_{i}(\lambda)$ and also $B=\sum_{i=1}^{k} y_{i}(S) E_{i}$ (Equation 1.2 and 1.3). Let us denote by $B_{i}$ and $S_{i}$ the restrictions of $B$ and $S$, respectively, to $E_{i} H=H_{i}$.

THEOREM 3.1. The operator $B$ has finite multiplicity, if and only if , the functions $y_{i}$ have $j_{i}\left(j_{i}<\infty\right)$ repetitions with respect to the spectral measure of $S_{i}$.

Also

$$
\max _{i} m u B_{i} \leq \sum_{i=1}^{k} m u B_{i} \leq \sum_{i=1}^{k} j_{i} m u S_{i} .
$$

Proof. From the definition of multiplicity, as the smallest number of generating elements, it follows that

$$
\max _{i} m u B_{i} \leq m u B \leq \sum_{i=1}^{k} m u B_{i} .
$$

Now, $B_{i}=y_{i}\left(S_{i}\right)$, hence the rest of the Theorem follows from Theorem 2.1. The problem of this section is reduced to the following

$$
H=\sum_{i=1}^{k} E_{i} H \text { where } E_{i} E_{j}=0 \text { if } i \neq j
$$

and $B_{i}=$ restriction $B$ to $E_{i} H$, where the multiplicity of $B_{i}$ is known. Now by decomposing each operator $B_{i}$ into sum of operators of uniform multiplicity we will have $H=\sum_{i=1}^{m} H_{i}$, where the spaces $H_{i}$ are mutually orthogonal, and $C_{i}=$ restriction of $B$ to $H_{i}$ is an operator of uniform multiplicity. We shall show how to compute $m u B$ from $m u C_{i}$ by reducing this case to the one studied in Theorem 2.2 , 
Denote the projection on $H_{i}$ by $F_{i}$. Let $E\left(B ; \alpha_{i}\right)$ be the maximal projection such that

$$
E\left(C_{i} ; \alpha_{i}\right)=E\left(B ; \alpha_{i}\right) F_{i}=0 .
$$

Such a projection exists by Zorn's Lemma. Finally let $\beta_{i}=$ $\sigma(B)-\alpha_{i}$. On $\beta_{i}$ the spectral measure of $C_{i}$ can vanish only when the spectral measure of $B$ vanishes. Now $E\left(B ; \bigcup_{i=1}^{m} \beta_{i}\right)=I$ because $\sum_{i=1}^{m} F_{i}=I$.

The set $\sigma(B)$ can be decomposed into disjoint sets $\gamma_{\text {, such that }}$

a. Each $\gamma_{j}$ is a subset of one of the sets $\beta_{j_{0}}$.

b. If $\gamma_{j} \cap \beta_{i} \neq \varphi$ then $\gamma_{j} \subset \beta_{i}$.

Assuming, for a moment, that this decomposition is given then

$$
m u B=\max _{j} m u\left(B \text { restricted to } E\left(B ; \gamma_{j}\right) H\right) .
$$

But the multiplicity of $B$ restricted to $E\left(B ; \gamma_{j}\right) H$ is

$$
\sum_{i \mid \gamma_{j} \subset \beta_{i}} m u\left(C_{i} \text { restricted to } E\left(B ; \gamma_{\jmath}\right) H_{i}\right)
$$

by Theorem 2.2 .

We shall show how to choose the sets $\gamma_{i}$ by an induction argument on the number $m$. Let $\gamma_{1}=\beta_{1}-\bigcup_{i \geq 2} \beta_{1} \beta_{i}$. This set (which might be void) satisfies conditions a and b. The rest of $\sigma(B)$ is

$$
\left(\bigcup_{i \geq 2} \beta_{1} \beta_{i}\right) \cup\left(\bigcup_{i \geq 2}\left(\beta_{i}-\beta_{1}\right)\right)
$$

In both sets there are only $m-1$ subsets and by induction there exists a decomposition.

\section{BIBLIOGRAPHY}

1. N. Dunford, and J. Schwartz, Linear Operators, Vol. II. to appear.

2. S. R. Foguel, Normal Operators of Finite Multiplicity. Communications on Pure and Applied Mathematics, Vol. XI, (1958), p. 297.

3. P. R. Halmos, Measure Theory. D. Van Nostrand, New York, 1950.

UNIVERSITY OF CALIFORNIA

BERKELEY, CALIFORNIA 


\section{PACIFIC JOURNAL OF MATHEMATICS}

\section{EDITORS}

David Gilbarg

Stanford University

Stanford, California

F. H. Brownell

University of Washington

Seattle 5, Washington
A. L. Whiteman

University of Southern Californıa Los Angeles 7, California

L. J. PaIge

University of California

Los Angeles 24, California

\section{ASSOCIATE EDITORS}
E. F. BECKENBACH
T. M. CHERRY
D. DERRY

E. HEWITT
A. HORN
L. NACHBIN
M. OHTSUKA

H. L. ROYDEN

M. M. SCHIFFER
E. SPANIER

E. G. STRAUS

F. WOLF

\section{SUPPORTING INSTITUTIONS}

\author{
UNIVERSITY OF BRITISH COLUMBIA \\ CALIFORNIA INSTITUTE OF TECHNOLOGY \\ UNIVERSITY OF CALIFORNIA \\ MONTANA STATE UNIVERSITY \\ UNIVERSITY OF NEVADA \\ NEW MEXICO STATE UNIVERSITY \\ OREGON STATE COLLEGE \\ UNIVERSITY OF OREGON \\ OSAKA UNIVERSITY \\ UNIVERSITY OF SOUTHERN CALIFORNIA
}

\author{
STANFORD UNIVERSITY \\ UNIVERSITY OF TOKYO \\ UNIVERSITY OF UTAH \\ WASHINGTON STATE COLLEGE \\ UNIVERSITY OF WASHINGTON \\ AMERICAN MATHEMATICAL SOCIETY \\ CALIFORNIA RESEARCH CORPORATION \\ HUGHES AIRCRAFT COMPANY \\ SPACE TECHNOLOGY LABORATORIES \\ NAVAL ORDNANCE TEST STATION
}

Mathematical papers intended for publication in the Pacific Journal of Mathematics should be typewritten (double spaced), and the author should keep a complete copy. Manuscripts may be sent to any one of the four editors. All other communications to the editors should be addressed to the managing editor, L. J. Paige at the University of California, Los Angeles 24, California.

50 reprints per author of each article are furnished free of charge; additional copies may be obtained at cost in multiples of 50 .

The Pacific Journal of Mathematics is published quarterly, in March, June, September, and December. The price per volume (4 numbers) is $\$ 12.00$; single issues, $\$ 3.50$. Back numbers are available. Special price to individual faculty members of supporting institutions and to individual members of the American Mathematical Society: $\$ 4.00$ per volume; single issues, $\$ 1.25$.

Subscriptions, orders for back numbers, and changes of address should be sent to Pacific Journal of Mathematics, 2120 Oxford Street, Berkeley 4, California.

Printed at Kokusai Bunken Insatsusha (International Academic Printing Co., Ltd.), No. 6, 2-chome, Fujimi-cho, Chiyoda-ku, Tokyo, Japan.

PUBLISHED BY PACIFIC JOURNAL OF MATHEMATICS, A NON-PROFIT CORPORATION

The Supporting Institutions listed above contribute to the cost of publication of this Journal, but they are not owners or publishers and have no responsibility for its content or policies. 


\section{Pacific Journal of Mathematics}

\section{Vol. 10, No. $2 \quad$ October, 1960}

Maynard G. Arsove, The Paley-Wiener theorem in metric linear spaces ........

Robert (Yisrael) John Aumann, Acceptable points in games of perfect

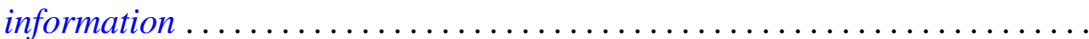

A. V. Balakrishnan, Fractional powers of closed operators and the semigroups generated by them ... . . . . . . . . . . . . . . . . . . . . . . . . . . . . 419

Dallas O. Banks, Bounds for the eigenvalues of some vibrating systems . . . . . 439

Billy Joe Boyer, On the summability of derived Fourier series . . . . . . . . . . . 475

Robert Breusch, An elementary proof of the prime number theorem with

remainder term ...................................

Edward David Callender, Jr., Hölder continuity of $n$-dimensional

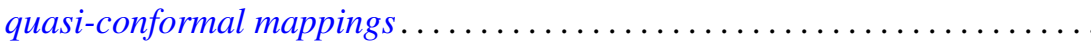

L. Carlitz, Note on Alder's polynomials ......................... 517

P. H. Doyle, III, Unions of cell pairs in $E^{3} \ldots \ldots \ldots \ldots \ldots \ldots \ldots \ldots \ldots \ldots \ldots \ldots . \ldots 21$

James Eells, Jr., A class of smooth bundles over a manifold . . . . . . . . . . . . 525

Shaul Foguel, Computations of the multiplicity function . . . . . . . . . . . . . . 539

James G. Glimm and Richard Vincent Kadison, Unitary operators in

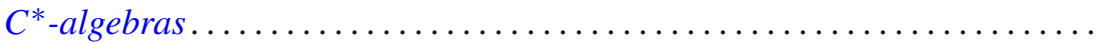

Hugh Gordon, Measure defined by abstract $L_{p}$ spaces . . . . . . . . . . . 557

Robert Clarke James, Separable conjugate spaces ....................

William Elliott Jenner, On non-associative algebras associated with bilinear forms

Harold H. Johnson, Terminating prolongation procedures

John W. Milnor and Edwin Spanier, Two remarks on fiber homotopy type .

Donald Alan Norton, A note on associativity . .

Ronald John Nunke, On the extensions of a torsion module.

Joseph J. Rotman, Mixed modules over valuations rings . . . . .

A. Sade, Théorie des systèmes demosiens de groupoï des . .

Wolfgang M. Schmidt, On normal numbers . .

661

Berthold Schweizer, Abe Sklar and Edward Oakley Thorp, The metrization of

statistical metric spaces

John P. Shanahan, On uniqueness questions for hyperbolic differential

equations

A. H. Stone, Sequences of coverings

Edward Oakley Thorp, Projections onto the subspace of compact operators

L. Bruce Treybig, Concerning certain locally peripherally separable spaces

Milo Wesley Weaver, On the commutativity of a correspondence and a

permutation

David Van Vranken Wend, On the zeros of solutions of some linear complex

differential equations. 